\title{
Expression of CD117, DOG-1, and IGF-1R in gastrointestinal stromal tumours - an analysis of 70 cases from 2004 to 2010
}

\author{
Joanna Kiśluk ${ }^{1}$, Justyna Zińczuk², Andrzej Kemona², Katarzyna Guzińska-Ustymowicz², Joanna Żurawska ${ }^{1}$, \\ Bogusław Kędra ${ }^{1}$
}

${ }^{1} 2^{\text {nd }}$ Department of General and Gastroenterological Surgery, Medical University of Bialystok, Bialystok, Poland

2Department of General Pathomorphology, Medical University of Bialystok, Bialystok, Poland

Gastroenterology Rev 2016; 11 (2): 115-122

DOI: $10.5114 / p g .2015 .52587$

Key words: gastrointestinal stromal tumours, CD117, DOG-1, IGF-1R.

Address for correspondence: Joanna Kiśluk PhD, $2^{\text {nd }}$ Department of General and Gastroenterological Surgery, Medical University of Bialystok, 24 A Sklodowskiej-Curie St, 15-276 Bialystok, Poland, phone: +48 8574859 35, fax: +48 8574859 88, e-mail: jk18@interia.pl

\begin{abstract}
Introduction: Determination of the type of mutations in gastrointestinal stromal tumours (GIST) plays a major role in assessing the risk of progression of the disease, and also allows determination of the clinical management and treatment. More accurate GIST diagnosis is possible by using simultaneously various types of antibodies to immunohistochemistry methods in routine procedures.

Aim: To evaluate the expression of CD117, DOG-1, and IGF-1R in patients with gastrointestinal stromal tumours, and analysis of the impact of the examined protein expression on patient survival with emphasis on specific recognition and prognostication of these tumours.

Material and methods: The protein expression was analyzed in 70 patients who had undergone surgical treatment for mesenchymal tumours of the gastrointestinal tract, using the immunohistochemical method.

Results: Positive expression of CD117, DOG-1, and IGF1R included $95.71 \%, 88.57 \%$ and $11.43 \%$ of study GISTs, respectively. Statistical analysis showed positive significant correlation between DOG-1 expression and histological type of tumour $(p=0.024)$. Analysis of overall survival curves of 70 GIST patients according to expression of CD117, DOG-1, and IGF1R did not show a tendency towards longer survival of patients with positive expression $(p>0.05)$.

Conclusions: Predictive factors determining the survival time of patients are strongly associated with morphological features of tumours. A thorough analysis of each case plays a key role in predicting survival time of patients and may be a clue in targeting the therapeutic procedure.
\end{abstract}

\section{Introduction}

Gastrointestinal stromal tumours (GIST) are defined as specific soft tissue tumours. They are derived from the multipotent precursor cells of Cajal, primarily responsible for the peristaltic movement of the tubular organs of the gastrointestinal tract [1]. The vast majority of these tumours (about 95\%) are characterised by the specific genetic profile of the mutation in the KIT (tyrosine kinase gene) and/or PDGFRA (platelet-derived growth factor receptor- $\alpha$ ) gene. KIT and PDGFRA are two highly homologous cell surface tyrosine kinase receptors for stem cell factor and platelet-derived growth factor alpha, respectively. Gain-of-function mutations in proto-oncogene c-KIT or PDGFRA have been thoroughly identified molecularly and also considered as the main factors that initiate carcinogenesis within Cajal cells.

Determination the type of mutations in stromal tumours plays a key role in assessing the risk of progression of the disease, and also allows determination of the clinical management and treatment. KIT receptor activating mutations occur in $60-70 \%$ of all GISTs, the most common among them being exon 11 (in-frame deletions or single nucleotide substitutions), exon 9 (duplications - mostly in intestinal GISTs), and exon 17 (internal tandem duplications - mostly gastric GISTs). PDGFRA mutations occur almost exclusively in gastric GISTs, most frequently in exon 18 . The routine histopathological di- 
agnostic procedures include haematoxylin-eosin staining to verify histological type of GIST tumours and immunohistochemistry verification with antibodies panel [1]. Positive expression of the KIT (CD117) antigen (epitope of the protein produced by the gene $K I T$ ) allows identification of GIST only in about $85 \%$ of cases (with a mutation in the KIT gene) [2]. A small percentage of GISTs $(3-5 \%)$ are only focally positive or negative, and these especially include gastric epithelioid GISTs with PDGFRA mutations. CD117-negative GISTs are also wild-type GISTs. For this reason, mesenchymal tumours should be precisely analysed for possible GIST neoplastic transformation, which allows the exclusion of possible false negative diagnostic errors. More accurate GIST diagnosis is possible by innovative DOG-1 antibody (discovered on GIST-1), which exhibits a positive protein expressed on the tumour cell surface regardless of the KIT/ PDGFRA mutation status [3]. DOG-1 protein is composed of the eight transmembrane regions and functions as a calcium ion-dependent chloride channel [4]. The gene encoding the DOG-1 is located on chromosome $11 \mathrm{q} 13$ (other names: FLJ10261, TMEM16A, ORAOV2) [3]. Similar to CD117, anoctamin-1 is also constitutively expressed in Cajal cells and in the vast majority of GISTs, including many KIT-negative GISTs, so that it supplements KIT in the positive identification of GIST. It has been shown that DOG-1 protein is characterised by high sensitivity (89\%) and specificity (94.8\%) relative to stromal tumour cells GIST [3, 4], and both cases are affected by the mutation of KIT/PDGFRA and WT-GIST.

Approximately $85 \%$ of paediatric GISTs and about $10-15 \%$ of adult GISTs do not show any mutations in the KIT and PDGFRA genes and are defined as KIT/ PDGFRA wild type (WT) [2]. In a small group of patients with WT-GIST there are other mechanisms suspected of malignant transformation initiators that have not yet been fully understood. Recent studies have suggested that besides KIT or PDGFRA mutations, aberrations of the insulin-like growth factor signalling pathway may play an important role in GIST tumorigenesis. This pathway consists of insulin-like growth factors (IGF1 and IGF2), IGF receptors (IGF1R and IGF2R), and IGF-binding proteins [5]. IGF1-R is a transmembrane protein belonging to the tyrosine kinases family of receptors that bind specifically to an IGF1 or IGF2 ligand. Activation of the IGF1-R receptor by combining the ligands stimulates intracellular signalling pathways (Ras-Raf-ERK/MAPK and $\mathrm{PI3K}-\mathrm{AKT} / \mathrm{mTOR}$ ) responsible for cell growth, proliferation, and apoptosis in normal tissues [6]. Overexpression of IGF1-R in many types of cancer, including breast, prostate [7, 8], and lung cancer [9], has been reported. Furthermore, it was found that the anti-apoptotic properties of IGF1-R allow tumour cells to resist the cytotox- ic properties of the drugs used in chemotherapy [7-9]. The ligand IGF1 functions primarily by activating the IGF1R, whereas IGF2 can act through either the IGF1R or through the insulin receptor $A$ isoform. Particularly high expression of IGF2 was reported in GIST and was correlated with a high rate of metastatic relapse in patients treated for a local disease [10]. Strong expression of IGF1-R has also been examined in stromal tumours [11, 12 ], where in the most common cases it was related to WT-GIST. The role of IGF1-R as a factor involved with the transformation of GIST unrelated to the genetic profile of KIT or PDGFRA requires more studies because knowledge of this area is still incomplete.

\section{Aim}

The aim of this study was to evaluate the expression of CD117, DOG-1, and IGF-1R in patients with GIST in correlation with clinico-pathological parameters. In this article we also analyse the impact of the examined protein expression on patient survival, with emphasis on specific recognition and prognostication of these tumours.

\section{Material and methods}

The study enrolled a group of 70 patients surgically treated for gastrointestinal stromal tumours in the Second Department of General and Gastroenterological Surgery at the Medical University of Bialystok (Poland) from the year 2004 to 2010. Resected GISTs derived from various localisations of the digestive track. Five-micrometre thick sections were cut from paraffin blocks and stained with haematoxylin-eosin $(H+E)$. Analysis of each GIST tumour included the following: diameter (cm), histological features of the tumours (epithelioid cell type, spindle cell type, mixed cell type), and mitotic index (the number of incorrect mitotic figures counted in 50 high-power fields).

\section{Immunohistochemical analysis}

Formalin-fixed and paraffin-embedded tissue specimens were cut on a microtome into 4- $\mu \mathrm{m}$ sections. The sections were deparaffinised in xylene and hydrated in alcohol. Antigens were exposed through citrate buffer heating $(\mathrm{pH}=6.0$ ) for $20 \mathrm{~min}$ and then cooled for at least $20 \mathrm{~min}$ at room temperature. After washing with distilled water and with PBS-buffer $(\mathrm{pH}=7.2)$ tissue sections were covered for five minutes with peroxidase blocking reagent (Novilink Polymer Detection System, Novocastra) to block endogenous peroxidase, followed by additional washing with the supplied buffer. Individual slides were then incubated with antibodies: anti-CD117 (mouse polyclonal anti-human CD117; corresponding to amino acids 927-942 of human Kit (c-Kit), Abcam; incuba- 
tion for 60 min at room temperature; dilution $1: 100$ ), DOG-1 (rabbit monoclonal anti-human DOG-1.1 clone K9, ThermoScientific; incubation $60 \mathrm{~min}$ at room temperature, dilution $1: 1$ - ready to use), anti-IGF-1R (mouse monoclonal anti-human IGF-1R, Abcam; incubation for $24 \mathrm{~h}$ at room temperature, dilution $1: 90$ ). The slides were washed three times with the buffer and then incubated with Post Primary Block (Novilink Polymer Detection System, Novocastra) for $30 \mathrm{~min}$ at room temperature after extensive washing with TRIS. Next, incubation was performed with Novolink Polymer (30 min and washing with TRIS; Novilink Polymer Detection System, Novocastra). The colour reaction was developed in DAB Chromogen (Novilink Polymer Detection System, Novocastra) according to the manufacturer's instructions. The sections were then counterstained with Meyer's haematoxylin, dehydrated, and mounted. The primary antibody solution in negative controls was substituted with a PBS-buffer.

Expression of CD117 and DOG-1 was determined using a semiquantitative method and assessed as positive (reaction visible in $>20 \%$ of tumour cells) or negative (lack of reaction, or reaction present in $<20 \%$ of cells). Expression of IGF-1R was also determined using a semiquantitative method based on gradation of intensity: negative (lack of reaction, or reaction present in $<50 \%$ of cells. Specimens with a diffused, high (+++; reaction visible in $76-100 \%$ of tumour cells), or moderate (++; reaction visible in $51-76 \%$ of tumour cells) immunostaining were considered as positive. Positive reactions were assessed in at least 500 cancer cells in each tissue specimen under a light microscope (400x).

Follow-up lasted through September 2014 (range: 2-155 months). Patients did not receive adjuvant or neoadjuvant therapy. Informed consent was obtained from each subject.

\section{Statistical analysis}

Statistical analysis was conducted using statistical software STATA 11.1. Analysis of the relationship between the studied subjects was performed using contingency table and $\chi^{2}$ test (two sample Wilcoxon rank-sum Mann-Whitney test and Kruskal-Wallis equality-of-population rank test). Relationships between expression of proteins were performed using Spearman rank correlation test. Kaplan-Meier survival analysis approach was employed to compare the overall survival rates of patients. A $p$-value $<0.05$ was considered statistically significant.

\section{Results}

Most of the GIST tumours ( $n=43,61.43 \%$ ) were located in the stomach. The second most frequent lo- cation related to the small intestine ( $n=15,21.43 \%$ ). Two tumours (2.4\%) were located in the retroperitoneal space and one in the duodenum. In further analysis they were included into the other locations $(n=3$, $4.29 \%)$. Most of the tumours had a diameter less than $2 \mathrm{~cm}(n=40,57.14 \%)$. Only three (4.29\%) tumours had a diameter excessing $10 \mathrm{~cm}$. Analysing the histological type of tumours, the most represented type was spindle cells tumour ( $n=43,61.43 \%)$. The second group included tumours with a predominance of epithelioid cells ( $n=17,24.29 \%)$. Analysis of the mitotic index (MI) showed that 49 tumours in the study group (70.00\%) had less than five atypical mitoses counted in $50 \mathrm{HPF}$. Higher IM > 5/50 HPF was observed in 21 (30.00\%) tumours from the entire group. Analysing the risk assessment of the progression and metastasis of tumours according to the AFIP classification [13] revealed that the vast majority of cases had a low $(40 / 57.14 \%)$ or a very low $(7 / 10.00 \%)$ risk of cancer progression.

Positive expression of CD117 was shown in 67 (95.71\%) of the tumours. In three tested tumours (4.29\%) the absence of CD117 expression was observed. A positive reaction was observed most commonly in the form of brown deposits localised in the plasma membrane or in the cytoplasm of GIST cells. Statistical analysis revealed no correlation between CD117 expression in primary GIST and clinico-pathological parameters. A spindle cell GIST with strong and diffuse cytoplasmic staining of CD117 is presented in Figure 1. Figure 2 shows a spindle cell GIST with strong and diffuse cytoplasmic and membrane staining of DOG-1.

The immunohistochemistry using DOG-1 antibody demonstrated positive expression in 62 (88.57\%) tumours, and in $8(11.43 \%)$ cases it showed a lack of reaction. Intense membrane and cytoplasmic staining of tumour cells in paraffin sections was observed. A statistically significant relationship was observed between DOG-1 expression and tumour histological type $(p=0.024)$. Most cases with positive DOG-1 expression were observed in spindle cells GISTs (40/70).

Expression of IGF-1R was positive in 8 (11.43\%) cases in the study group. The strongest colour reaction was seen in 5 cases (5.95\%), where more than $75 \%$ specifically positive cells were found. The analysis of the distribution of IGF1R expression depending on the tumour location, size and histological type, mitotic index, and risk of progression revealed no significant correlation $(p>0.05)$.

In addition, in statistical analysis we did not observe any significant correlation between IGF1R expression and CD117 and DOG-1 $(p>0.05)$. Detailed characteristics of the study group and distribution of expression is shown in Table I. 


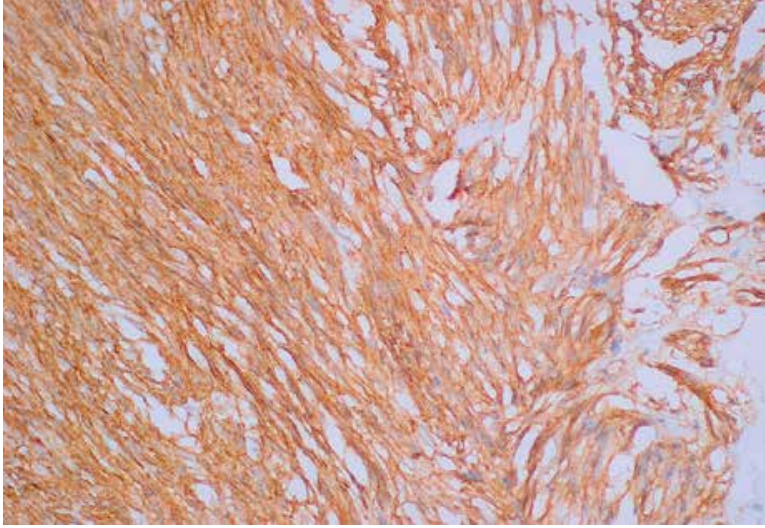

Figure 1. Spindle cells GIST with strong and diffuse cytoplasmic staining of CD117 (c-kit) (400x)

Patients overall survival range was 2-155 months. At the time of analysis, 12 patients had died (it has not been determined whether the death was associated with the disease). There was no statistically significant dependence of survival time according to the expression of CD117, DOG-1, and IGF1R ( $p>0.05)$. Comparison of survival curves over the course of the remaining antibodies did not indicate significant differences between the positive expression or its absence $(p>0.05)$. During analysis, IGF1R survival curves showed a tendency to longer survival in patients with positive expression ( $p=0.079$ ) but it was not statistically significant in our small group of patients. Figures 3-5 show the dependence of the probability of survival of patients with GIST to CD117, DOG-1, and IGF1R expression, respectively.

\section{Discussion}

Gastrointestinal stromal tumours are a group at high risk of malignant transformation, presumably derived from stem cells and the specialised interstitial cells of Cajal (ICCS). In our study group of 70 patients surgically treated at the Second Department of General and Gastroenterological Surgery of the Medical University of Bialystok on the basis of positive expression of CD117 antigen diagnosis of GIST was approved in $63(95.71 \%)$ patients. As it turned out, the result of KIT expression was not sensitive enough for all cases. Current research on the GIST genetic profile demonstrates that mutation of the proto-oncogene $\mathrm{C}-\mathrm{KIT}$, besides mutation in the tyrosine kinase KIT gene, is also manifested by changes in PDGFRA. Studies using molecular biology techniques have identified that both genes encode highly homologous transmembrane glycoprotein of the same name. These glycoproteins are of a type III receptor tyrosine kinase family. Its overexpression is

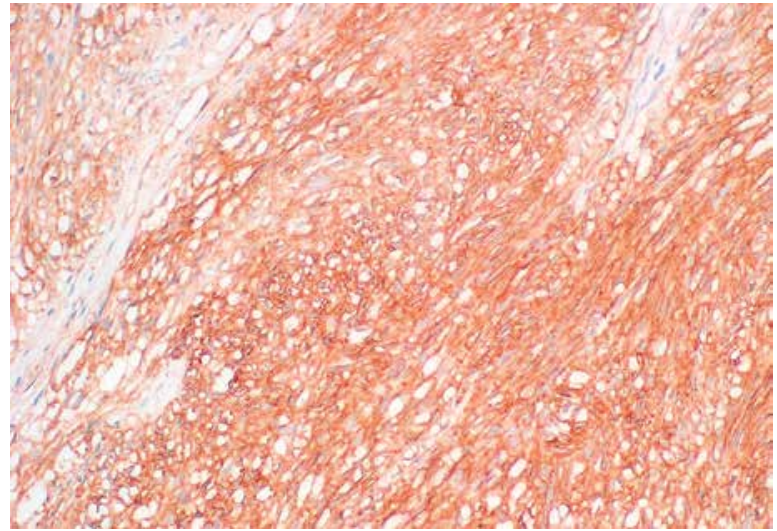

Figure 2. Spindle cells GIST with strong and diffuse cytoplasmic and membrane staining of DOG-1 (400x)

associated with somatic mutations that lead to a constant and ligand-independent autophosphorylation of the kinase receptor KIT or PDGFRA. This leads to changes in conformation of the receptor and the intracellular signal transduction pathways, such as PI3K/AKT, MAPK, and STAT $[14,15]$. Overexpression of these receptors is highly specific for GIST and is the most important criterion in the diagnosis and classification of microscopic treatment of patients to highly specific small-molecule tyrosine kinase inhibitor [16].

Diagnostic standards of GISTs suggest that histopathological diagnosis of GIST should be based on immunohistochemically positive expression of CD117 or DOG-1 [17, 18]. Although these antibodies are generally used interchangeably, the positive expression has a slightly different biological dimension $[19,20]$. The physiological function of DOG-1 is not fully understood. West et al. [21], on the basis of microarray gene analysis of DOG-1 and the results of in situ hybridisation and immunohistochemistry, suggests that this marker is part of the ion transporter family as yet unclassified. The presence of a strong expression of DOG-1 was not exactly specified. This protein is either involved in the signal transduction pathway for the receptor kinase type III, or a random marker specific for GIST, unrelated to the mutation status [22]. Regardless of the physiological role of DOG-1, the fact that this marker is correlated with patient survival is important in the stromal tumour oncogenesis. Kang et al. [23] in his research showed that negative DOG-1 expression is an independent prognostic factor for shorter overall survival $(p=0.002)$. Our results partially confirm this thesis, indicating that the positive immunoreactivity of GIST cells in the direction of DOG-1 could result in longer survival, but the low number of patients in the group does not give statistically significant results. Despite this, our results show positive statistical 


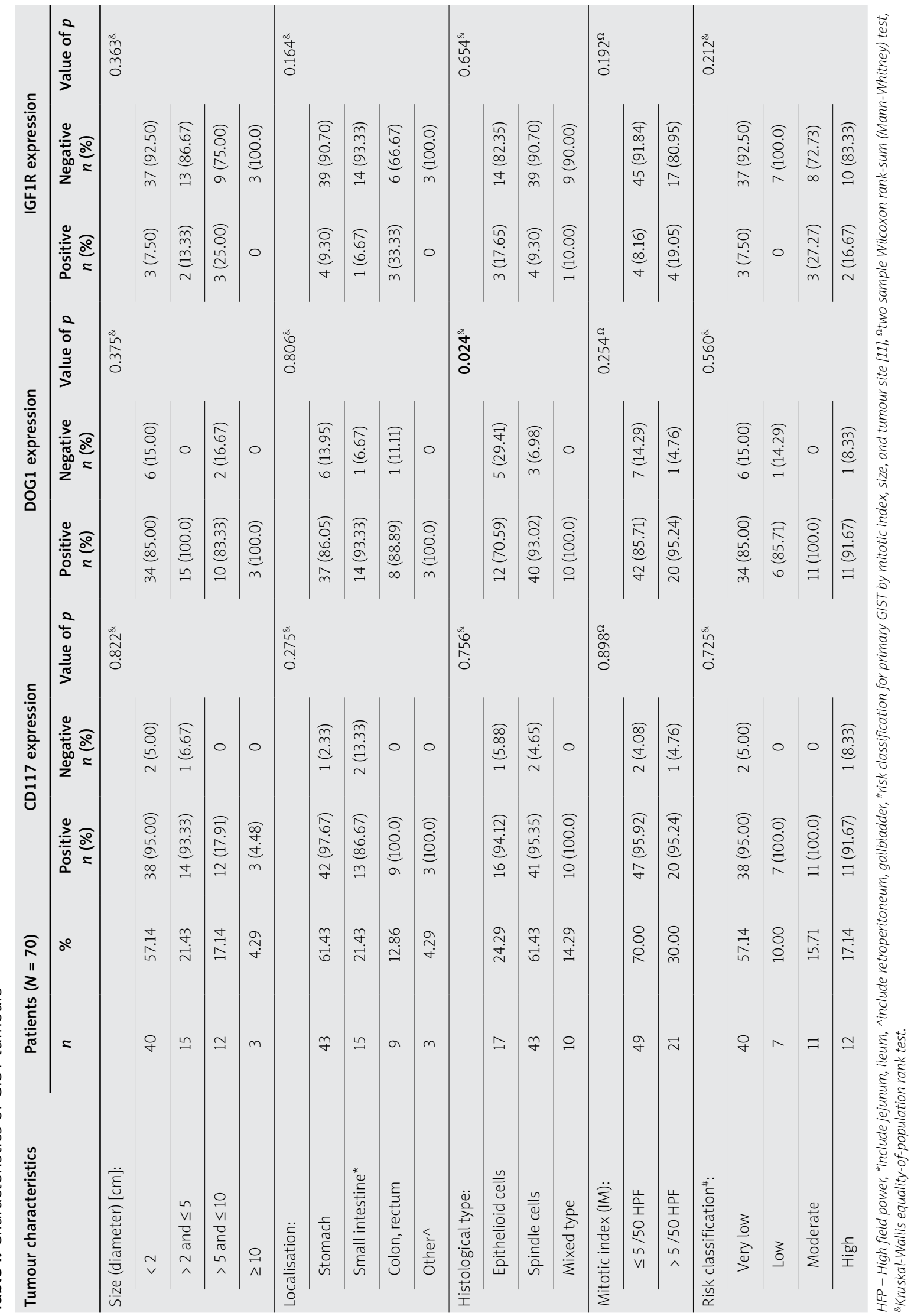




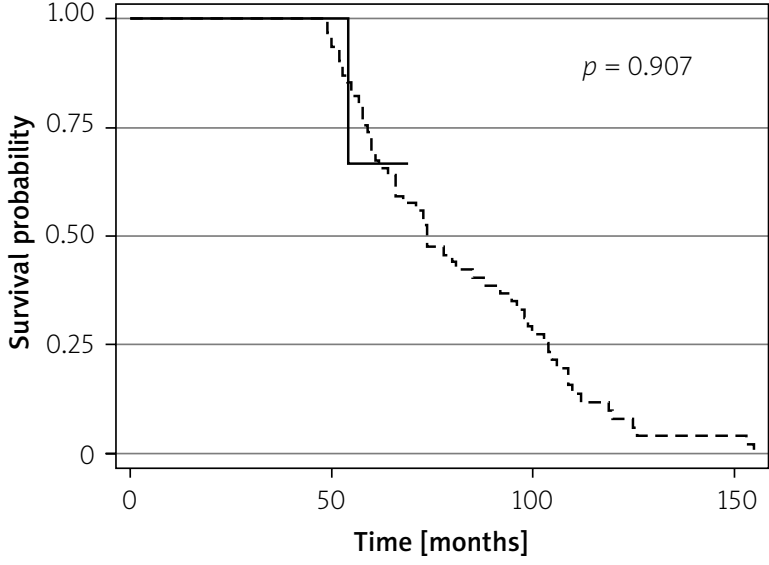

Number at risk:

$\begin{array}{lcccc}C D 117- & 3 & 3 & 0 & 0 \\ \mathrm{CD} 117+ & 67 & 59 & 15 & 2\end{array}$

- - - CD117 positive - CD117 negative

Figure 3. Overall survival curves of GIST patients according to expression of CD117

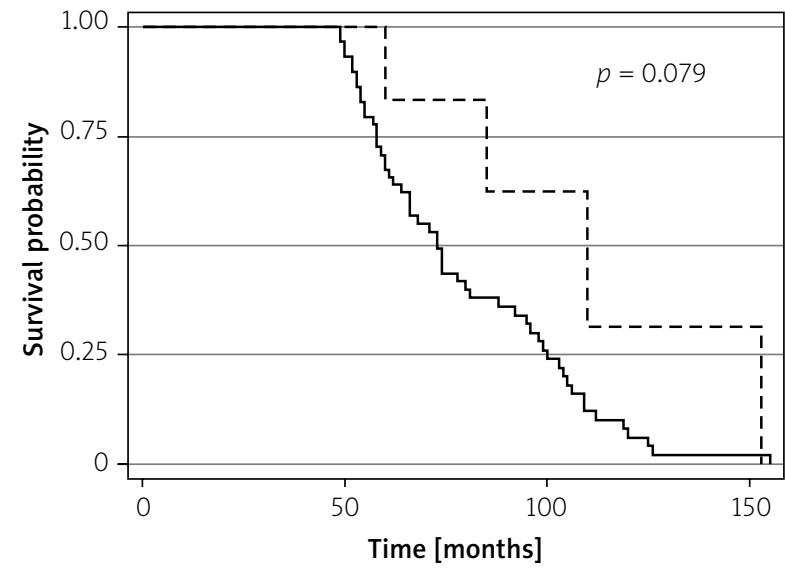

Number at risk:

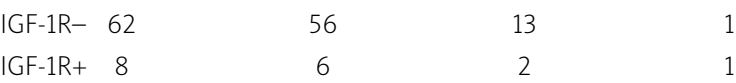

- - - IGF1R positive —IGF1R negative

Figure 5. Overall survival curves of GIST patients according to expression of IGF-1R

correlation between DOG-1 expression and histological type of tumour ( $p=0.024)$. Some data suggest that histological type may also impact the prognosis of GIST patients. Singer et al. [24], in a report of 48 patients, reported that the 5-year recurrence-free survival rate was significantly higher among patients with spindle cell as compared to epithelioid or mixed histology. However, others report a prognostic influence of the degree of cellularity but not histologic subtype [25].

Studies on large populations of patients with GIST have shown that approximately $1-2 \%$ of GIST tumours

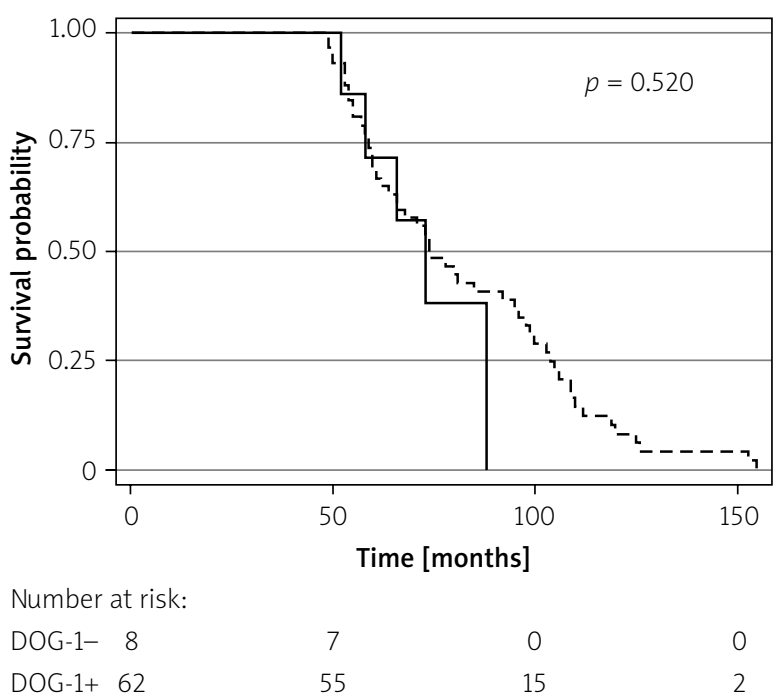

- - - DOG1 positive _ DOG1 negative

Figure 4. Overall survival curves of GIST patients according to expression of DOG-1

are CD117 and DOG-1 negative. Lopes et al. [4] and Novelli et al. [26] documented, respectively, $0.9 \%$ and $1.6 \%$ of such cases. This GISTs occurring in adults who do not harbour KIT or PFGFRA mutations are defined as KIT/ PDGFRA wild type (WT).

In 2008 Lorincz et al. [27] identified and characterised precursors of ICCS as phenotype IGF1R+, $\mathrm{KIT}^{\text {+low, }}$ $\mathrm{CD}_{4}{ }^{+}, \mathrm{CD} 4^{+}$, and Insr ${ }^{+}$. This study suggested also that differentiation of ICC precursors into mature ICCS is driven by IGF1 and IGF1R pathway and may play an important role in GIST pathogenesis and development. In the same year, Tarn et al. [11] suggested a significant genetic defect in the IGF-1R gene as another means of tumorigenesis in GISTs, which is manifested by overexpression of the IGF-1R protein. Furhter studies have shown overexpression of IGF1R at the MRNA and protein level without IGF1R genomic amplification, which suggests that another mechanism may be involved [12, 28]. The molecular basis of IGF1R overexpression in WT GISTs has not yet been explained and mechanisms other than copy number abnormalities or activating mutations are responsible [29].

Expression of IGF-1R in the present study using immunohistochemistry methods showed a positive reaction in $8(11.43 \%)$ cases. Despite our group including only 70 patients, the proportion of positive tumours seems to be similar to latest results of Lasota, where, during examination of a large number (1078) of GIST cases, $8 \%$ were IGF1R positive [30]. In our study, all IGF1R+ GISTs have more than $50 \%$ stain-positive tumour cells. One of them was negative for DOG-1 and CD117, with characteristic morphological features of GIST cells. We observed no sta- 
tistically significant correlation between pathological features and IGF1R expression ( $p>0.05)$. Analysis of overall survival curves of 70 GIST patients according to expression of IGF1R (Figure 5) shows a tendency towards longer patients survival with positive expression $(p=0.079)$. Valadão et al. [31] presented conflicting results - in his research on 59 patients with GIST, higher IGF1R expression did not affect progression-free and overall survival. There was also a statistically significant association between IGF1R expression and type of response to imatinib treatment ( $p=0.05)$ - higher IGF1R expression was related to lower objective response. In both outcomes there is the need to verify the results on a larger group of patients.

Braconi et al. [32] showed that overexpression of the IGF-1R receptor with increased amount of the ligand (IGF1 and IGF2) correlate with disease recurrence in patients with diagnosed and treated GIST. IGF pathway disruption and IGF1R inhibition are currently investigated as rational therapeutic targets in both mutant GIST and wild-type GIST. Research in this area suggests that examination of IGF1R expression is important in determining the response.

Overexpression of IGF1R is one of the alternative drivers responsible for acquired imatinib resistance [11, $33,34]$. Advanced clinical trials over the mechanism of action of small molecule inhibitors of IGF-1 receptor (NVP-AEW541) or interfering RNA showed induction of cytotoxicity in GIST cell lines in vitro [34]. The cytotoxic effect was observed both in imatinib-resistance mutant GIST cell lines and imatinib-sensitive cells. This proves that IGFR-1 pathway is an important driver of oncogenesis, and inhibition can potentially be used as primary therapy in wild-type GIST and as a complementary therapy in mutant GISTs.

Subsequent research proved that IGFR1 expression in WT GISTs is associated with SDHB (succinate dehydrogenase B) deficiency $[35,36]$. New therapeutic guidelines for imatinib-resistance WT GISTs include emerging therapeutic targets and potential agents related with IGF1R inhibition [33]. A phase Ib study of monoclonal antibody R1507 (in preclinical studies and limited clinical setting) and a phase II trial of linsitinib demonstrated promising results [37, 38].

\section{Conclusions}

Many study results show that the survival time of patients with stromal tumours depends on numerous factors. The presence or absence of specific morphological features of the tumour affects directly the prognosis of the patient. It is also the basis for classifying or excluding patients from a drug program, using inhibitors of tyrosine kinase inhibitors (Imatinib, Sunatinib) or second therapeutic strategy using PI3K/AKT/mTOR inhibitors, heat shock protein 90 inhibitors, IGF1R inhibitors, and immune therapy. Predictive factors determining the survival time of patients are strongly associated with the morphological features of the tumours. A thorough analysis of each case plays a key role in predicting survival time of patients and may be a clue in targeting the therapeutic procedure.

\section{Conflict of interest}

The authors declare no conflict of interest.

\section{References}

1. Miettinen M, Lasota J. Gastrointestinal stromal tumors: review on morphology, molecular pathology, prognosis, and differential diagnosis. Arch Pathol Lab Med 2006; 130: 1466-78.

2. Lasota J, Miettinen M. Clinical significance of oncogenic KIT and PDGFRA mutations in gastrointestinal stromal tumours. Histopathology 2008; 53: 245-66.

3. Miettinen M, Wang ZF, Lasota J. DOG1 antibody in the differential diagnosis of gastrointestinal stromal tumors: a study of 1840 cases. Am J Surg Pathol 2009; 33: 1401-8.

4. Lopes LF, West RB, Bacchi LM, et al. DOG1 for the diagnosis of gastrointestinal stromal tumor (GIST): comparison between 2 different antibodies. Appl Immunohistochem Mol Morphol 2010; 18: 333-7.

5. Pollak $M$. Insulin and insulin-like growth factor signalling in neoplasia. Nat Rev Cancer 2008; 8: 915-28.

6. $\mathrm{Yu} \mathrm{H}$, Rohan T. Role of the insulin-like growth factor family in cancer development and progression. J Natl Cancer Inst 2000; 92: 1472-89.

7. Hellawell GO, Turner GD, Davies DR, et al. Expression of the type 1 insulin-like growth factor receptor is up-regulated in primary prostate cancer and commonly persists in metastatic disease. Cancer Res 2002; 62: 2942-50.

8. Jones HE, Goddard L, Gee JM, et al. Insulin-like growth factor-I receptor signalling and acquired resistance to gefitinib (ZD1839; Iressa) in human breast and prostate cancer cells. Endocr Relat Cancer 2004; 11: 793-814.

9. Warshamana-Greene GS, Litz J, Buchdunger E, et al. The insulin-like growth factor-I receptor kinase inhibitor, NVP-ADW742, sensitizes small cell lung cancer cell lines to the effects of chemotherapy. Clin Cancer Res 2005; 11: 1563-71.

10. Agaram NP, Laquaglia MP, Ustun B, et al. Molecular characterization of pediatric gastrointestinal stromal tumors. Clin Cancer Res 2008; 14: 3204-15.

11. Tarn C, Rink L, Merkel E, et al. Insulin-like growth factor 1 receptor is a potential therapeutic target for gastrointestinal stromal tumors. Proc Natl Acad Sci U S A 2008; 105: 8387-92.

12. Janeway KA, Zhu MJ, Barretina J, et al. Strong expression of IGF1R in pediatric gastrointestinal stromal tumors without IGF1R genomic amplification. Int J Cancer 2010; 127: 2718-22.

13. Miettinen $M$, Lasota J. Gastrointestinal stromal tumors: pathology and prognosis at different sites. Semin Diagn Pathol 2006; 23: 70-83.

14. Rutkowski P, Andrzejuk J, Bylina E, et al. What are the current outcomes of advanced gastrointestinal stromal tumors: who 
are the long-term survivors treated initially with imatinib? Med Oncol 2013; 30: 765 .

15. Hirota S, Isozaki K, Moriyama Y, et al. Gain-of-function mutations of c-kit in human gastrointestinal stromal tumors. Science 1998; 279: 577-80

16. Kern A, Görgens H, Dittert DD, et al. Mutational status of KIT and PDGFRA and expression of PDGFRA are not associated with prognosis after curative resection of primary gastrointes tinal stromal tumors (GISTs). J Surg Oncol 2011; 104: 59-65.

17. Tan CB, Zhi W, Shahzad G, Mustacchia P. Gastrointestinal stromal tumors: a review of case reports, diagnosis, treatment, and future directions. ISRN Gastroenterol 2012; 2012: 595968

18. Kang GH, Srivastava A, Kim YE, et al. DOG 1 and PKC- $\theta$ are useful in the diagnosis of KIT-negative gastrointestinal stromal tumors. Mod Pathol 2011; 24: 866-75.

19. Ríos-Moreno MJ, Jaramillo S, Pereira Gallardo S, et al. Gastrointestinal stromal tumors (GISTs): CD117, DOG-1 and PKC $\theta$ expression. Is there any advantage in using several markers? Pathol Res Pract 2012; 208: 74-81.

20. Miwa S, Nakajima T, Murai Y, et al. Mutation assay of the nove gene DOG1 in gastrointestinal stromal tumors (GISTs). J Gastroenterol 2008; 43: 531-7.

21. West RB, Corless CL, Chen X, et al. The novel marker, DOG1, is expressed ubiquitously in gastrointestinal stromal tumors irrespective of KIT or PDGFRA mutation status. Am J Patho 2004; 165: 107-13.

22. Heinrich MC, Corless CL, Demetri GD, et al. Kinase mutations and imatinib response in patients with metastatic gastrointestinal stromal tumor. J Clin Oncol 2003; 21: 4342-9.

23. Kang YN, Jung HR, Hwang I. Clinicopathological and immunohistochemical features of gastointestinal stromal tumors. Cancer Res Treat 2010; 42: 135-43.

24. Singer S, Rubin BP, Lux ML, et al. Prognostic value of KIT mutation type, mitotic activity, and histologic subtype in gastrointestinal stromal tumors. J Clin Oncol 2002; 20: 3898-905.

25. Reith JD, Goldblum JR, Lyles RH, Weiss SW. Extragastrointesti nal (soft tissue) stromal tumors: an analysis of 48 cases with emphasis on histologic predictors of outcome. Mod Pathol 2000; 13: 577-85.

26. Novelli M, Rossi S, Rodriguez-Justo $M$, et al. DOG1 and CD117 are the antibodies of choice in the diagnosis of gastrointestinal stromal tumours. Histopathology 2010; 57: 259-70.

27. Lorincz A, Redelman D, Horváth VJ, et al. Progenitors of interstitial cells of cajal in the postnatal murine stomach. Gastroenterology 2008; 134: 1083-93.

28. Pantaleo MA, Astolfi A, Di Battista M, et al. Insulin-like growth factor 1 receptor expression in wild-type GISTs: a potential novel therapeutic target. Int J Cancer 2009; 125: 2991-4.

29. Italiano A, Chen J, Zhang L, et al. Patterns of deregulation of insulin growth factor signalling pathway in paediatric and adult gastrointestinal stromal tumours. Eur J Cancer 2012; 48: 3215-22.

30. Lasota J, Wang Z, Kim SY, et al. Expression of the receptor for type I insulin-like growth factor (IGF1R) in gastrointestinal stromal tumors: an immunohistochemical study of 1078 cases with diagnostic and therapeutic implications. Am J Surg Pathol 2013; 37: 114-9.
31. Valadão M, Braggio D, Santos AF, et al. Involvement of signaling molecules in the prediction of response to imatinib treatment in metastatic GIST patients. J Surg Res 2012; 178: 288-93.

32. Braconi C, Bracci R, Bearzi I, et al. Insulin-like growth factor (IGF) 1 and 2 help to predict disease outcome in GIST patients. Ann Oncol 2008; 19: 1293-8.

33. Songdej N, von Mehren M. GIST treatment options after tyrosine kinase inhibitors. Curr Treat Options Oncol 2014; 15: 493-506.

34. Patel S. Exploring novel therapeutic targets in GIST: focus on the PI3K/Akt/mTOR pathway. Curr Oncol Rep 2013; 15: 386-95.

35. Belinsky MG, Rink L, Flieder DB, et al. Overexpression of insulin-like growth factor 1 receptor and frequent mutational inactivation of SDHA in wild-type SDHB-negative gastrointestinal stromal tumors. Genes Chromosomes Cancer 2013; 52: 214-24.

36. Belinsky MG, Rink L, von Mehren M. Succinate dehydrogenase deficiency in pediatric and adult gastrointestinal stromal tumors. Front Oncol 2013; 3: 117.

37. Mahadevan D, Sutton GR, Arteta-Bulos R, et al. Phase 1 b study of safety, tolerability and efficacy of R1507, a monoclonal antibody to IGF-1R in combination with multiple standard oncology regimens in patients with advanced solid malignancies. Cancer Chemother Pharmacol 2014; 73: 467-73.

38. Pessetto ZY, Weir SJ, Sethi G, et al. Drug repurposing for gastrointestinal stromal tumor. Mol Cancer Ther 2013; 12: 1299-309.

Received: 21.01.2015

Accepted: 8.03.2015 